\title{
Kasuistiken
}

Rechtsmedizin 2020 30:253-259

https://doi.org/10.1007/s00194-020-00398-w

Online publiziert:25. Mai 2020

(C) Der/die Autor(en) 2020

\author{
I. Wirth $\cdot$ R. Schulz ${ }^{2}$ S. Schmidt ${ }^{2} \cdot$ A. Schmeling ${ }^{2}$ \\ 'Berlin, Deutschland \\ ${ }^{2}$ Institut für Rechtsmedizin, Universitätsklinikum Münster, Münster, Deutschland
}

\section{Schrubber als Tatwaffe}

\section{Tötung des 86-jährigen Ehemanns durch die gleichaltrige Ehefrau}

\section{Einleitung}

Eine stumpfe Gewalt ist charakterisiert durch eine flächige oder kantige Einwirkung auf den menschlichen Körper. Dabei lassen sich 3 grundsätzliche Verletzungsmechanismen unterscheiden: Neben der Kollision, bei der 2 bewegte Objekte zusammenstoßen, kann der sich bewegende Mensch auf ein ruhendes Widerlager treffen (z.B. beim Sturz) oder umgekehrt ein in Bewegung befindlicher Gegenstand auf den Körper einwirken. Zu dem letztgenannten Geschehen zählen Schläge mit den Fäusten und mit Gegenständen (z.B. Knüppel, Hämmer), Fußtritte sowie geworfene und herabfallende Gegenstände (z.B. Pflastersteine, Dachziegel). Die vielfältigen, zur Verletzung führenden Bewegungsabläufe sind ein Grund für die außerordentliche morphologische Variabilität der Folgen stumpfer Gewaltwirkung. Konkret wird das entstehende Verletzungsmuster von physikalischen Gewaltparametern, wie Masse und Geschwindigkeit, Einwirkungswinkel und Wirkfläche des verletzenden Gegenstands, bestimmt. Zugleich beeinflussen die anatomische Beschaffenheit der betroffenen Körperstelle und die Reaktion des Organismus auf die schädigende Einwirkung das Befundmuster. Nicht zuletzt resultiert durch Überlagerung von Mehrfachverletzungen eine Modifikation des morphologischen Erscheinungsbildes.

Typische Verletzungsarten an der Körperoberfläche sind Hautabschürfung, Hämatom und die Quetsch-Riss-

Frau Prof. Dr. med. Heidi Pfeiffer zum 60. Geburtstag gewidmet.
Wunde. Ebenso sind offene und auch manche geschlossenen Frakturen äußerlich feststellbar. Allerdings vermittelt die äußere Besichtigung des Körpers keinen vollständigen Überblick über Art und Schwere aller Verletzungen. Vielmehr können in der Tiefe der Gewebe und an den inneren Organen schwere bis todeswürdige Schädigungen vorhanden sein. Abhängig vom Ausmaß der stumpfen Gewalteinwirkung kommen verschiedene Todesursachen in Betracht. Der Tod kann infolge erheblicher Organschäden (Zerreißung, Zermalmung), sekundärer Folgen der Verletzungen (Blutung, Aspiration, Kreislaufschock, Fettembolie, Luftembolie) oder schwerwiegender Komplikationen (z.B. Wundinfektion, Thromboembolie) eintreten.

Stumpfe Gewalt ist die dominierende Form physischer Gewalt bei körperlichen Auseinandersetzungen in häuslichen Gemeinschaften. Die Angriffe richten sich sowohl gegen kindliche als auch gegen erwachsene Opfer. In heterosexuellen Partnerschaften sind es weit überwiegend Frauen, die angegriffen werden, jedoch sind auch Männer in einer nennenswerten Häufigkeit von partnerschaftlicher Gewalt betroffen [3]. Die Täterinnen schlagen mit der flachen Hand, der Faust oder mit Gegenständen, treten barfuß oder mit einem Schuh am Fuß, beißen und kratzen, ziehen an den Haaren, stoßen ihr Opfer zu Boden oder eine Treppe hinunter. Strafrechtlich reicht das Tatspektrum auch bei männlichen Opfern von Mord und Totschlag über Körperverletzungs- und Sexualdelikte bis zu Bedrohung, Stalking, Nötigung und Freiheitsberaubung [2].
Nachfolgend wird über einen tödlich endenden Fall häuslicher Gewalt berichtet, bei dem nicht nur das Alter der Beteiligten, sondern auch das verwendete Tatwerkzeug ungewöhnlich ist.

\section{Falldarstellung}

\section{Vorgeschichte}

Über den Notruf ging bei der Polizei am Spätnachmittag des 25.12.2018 die Mitteilung ein, dass ein älterer Mann in der Wohnung gestürzt sei und sich nicht mehr bewegen könne. Zeitgleich mit den entsandten Polizeibeamten trafen die ebenfalls alarmierten Rettungskräfte am Ereignisort ein. Sie fanden einen leblosen Mann, der in der Küche auf dem Fußboden lag. Die unbekleideten Körperpartien ließen zahlreiche Hämatome erkennen; Kopfhaut und linkes Auge waren stark geschwollen. In der Umgebung des Mannes befanden sich am Küchenmobiliar diverse Blutspritzer und auf dem Fußboden unregelmäßige, blutige Wischspuren. Die Ehefrau gab an, sie habe sich am Nachmittag im Wohnzimmer aufgehalten. Von dort habe sie einen Schrei ihres Mannes und einen Knall wahrgenommen. Daraufhin sei sie in die Küche gegangen und habe ihren Mann am Boden liegend vorgefunden.

Die angeforderte Notärztin stellte den Tod des Mannes fest. Bei der anschließenden kriminalistischen Leichenuntersuchung bestätigte sich der sofort geäußerte Verdacht, dass die festgestellten multiplen Verletzungen nicht die Folgen eines Sturzes zu ebener Erde sein konnten. Von der Polizei wurde am Leichenfundort ein Schrubber als mögliches Tat- 


\section{Kasuistiken}

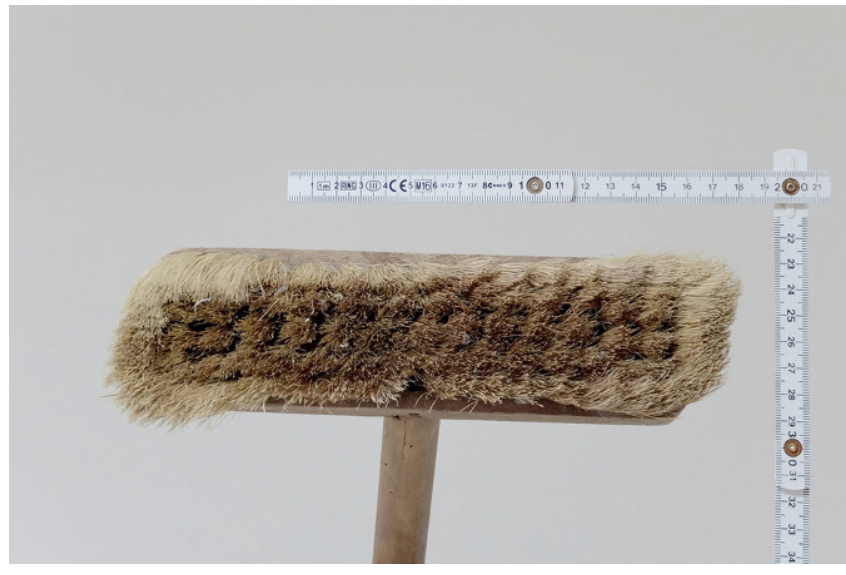

Abb. $1<$ Borstenbüschel des als Tatwerkzeug verwendeten Schrubbers



Abb. $2<$ Lange Seite des Bürstenkörpers mit Borstenbüscheln

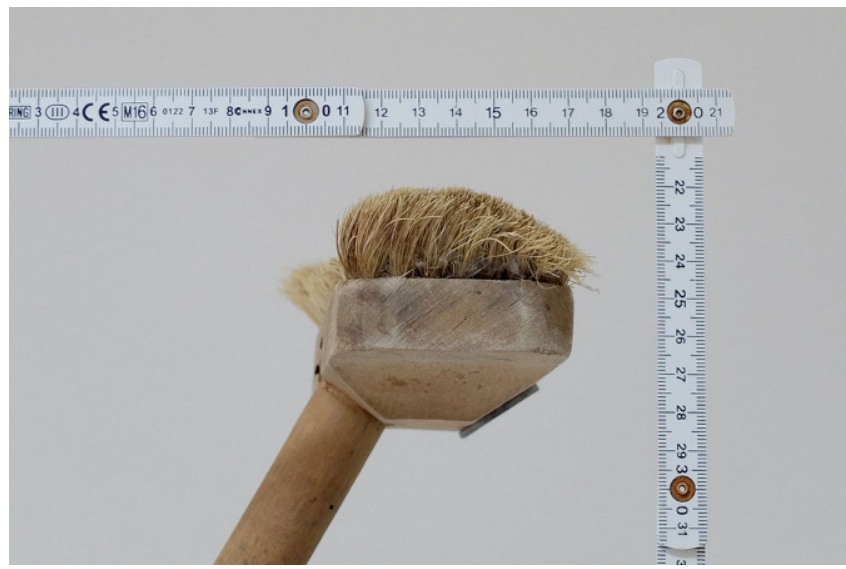

Abb. $3<$ Kurze Seite des Bürstenkörpers mit Borstenbüscheln

werkzeug sichergestellt (• Abb. 1, 2, 3). Gegen die Ehefrau wurde ein Ermittlungsverfahren wegen Totschlags gemäß $\S 212$ StGB eingeleitet.

\section{Obduktion und Zusatz- untersuchungen}

Bei der gerichtlichen Leichenöffnung am 26.12.2018 fanden sich deutliche Zeichen vielfacher Gewalteinwirkungen gegen den Körper. Die linke obere Gesichtshälfte wies eine im Durchmesser bis $10 \mathrm{~cm}$ große, violett vertrocknete oberflächliche Hautabschürfung auf, die von der Schläfe bis in die linke Augenregion reichte. Über dem linken Unterkieferast und in der angrenzenden Wangenregion zeigten sich zahlreiche, angedeutet regelmäßig verteilte, $z$. T. längs gestellte, z. T. bogig konfigurierte, strichförmige, bis $1 \mathrm{~cm}$ lange oberflächliche Hautde- fekte. Das verletzte Areal imponierte insgesamt als rechteckig konturierte Fläche (• Abb. 4). An der Beugeseite des rechten Oberarms waren zahlreiche, stellenweise zusammenstehende, im Durchmesser bis $3 \mathrm{~mm}$ große, violette Hautverfärbungen feststellbar. An der Außenseite des rechten Oberarms lag eine Vielzahl kratzerartiger, bis $3 \mathrm{~cm}$ langer, strichförmiger, bräunlich verschorfter Oberhautdefekte (• Abb. 5). Weitere gruppiert angeordnete Verletzungen befanden sich im Nacken, wo sich zahlreiche, im Durchmesser bis $3 \mathrm{~mm}$ große, violette Hautverfärbungen und etwa gleichgroße, braun-violett vertrocknete oberflächliche Hautdefekte verteilten (-Abb. 6). In der linken hinteren Schulterregion fand sich eine $20 \mathrm{~cm} \times 15 \mathrm{~cm}$ große, braun-violette Hautverfärbung. Innerhalb der verfärbten Fläche traten mehrere rechtwinklige, tief blau-violette Hautverfärbungen mit einer Kantenlänge von etwa $5 \mathrm{~cm}$ hervor (- Abb. 7).

Über die geformten Verletzungen hinaus fanden sich zahlreiche, mehrheitlich flächenhafte Hautunterblutungen und vielfache Hautabschürfungen an Kopf, Rumpf und Gliedmaßen. In beiden Stirn-Scheitel-Regionen waren große Wundtaschen nachweisbar. Bei der Präparation der Extremitäten stellten sich ausgedehnte Wundtaschen am linken Arm und am rechten Bein dar. Auch an der linken Schulter und in der linken Gesäßregion zeigten sich kräftig eingeblutete Wundtaschen im Unterhautfettgewebe.

An der Schleimhaut von Ober- und Unterlippe bestanden Zahnkonturabdrücke. Die Nasenwurzel wies eine im Durchmesser bis $1,5 \mathrm{~cm}$ große, sternförmige Hautaufreißung und an korrespondierender Stelle eine Nasenbeinfraktur auf. Weiterhin lagen kräftig umblutete Frakturen beider oberer Schildknorpelhörner und gleichfalls kräftig unterblutete Rippenserienfrakturen beiderseits vor.

Als Zeichen des Blutverlusts waren spärliche Totenflecke, Blutarmut der inneren Organe und kleinfleckige subendokardiale Blutungen in der linken Herzkammer vorhanden. 
Die makroskopisch festgestellten krankhaften Organveränderungen waren nicht geeignet, den Todeseintritt $\mathrm{zu}$ erklären. Auch die umfangreichen histologischen Untersuchungen der inneren Organe ergaben keine Hinweise auf schwergradige vorbestehende Erkrankungen oder auf ein todesursächlich konkurrierendes Grundleiden. Die mikroskopisch untersuchten Proben von Haut und Weichgeweben zeigten das Bild ganz überwiegend frischerer, bis wenige Stunden alter Einblutungen, einzelne Gewebsproben auch mit Zeichen wenige Tage alter Einblutungen bei bindegewebiger Organisation. Es ergab sich kein Hinweis auf eine Fettembolie.

Bei den toxikologisch-chemischen Untersuchungen von Herzblut, Mageninhalt und Urin wurden Metaboliten des $\beta$-Rezeptoren-Blockers Metoprolol im Urin nachgewiesen. Die Analysen erbrachten keine Anhaltspunkte für die Aufnahme von zentralwirksamen Arzneistoffen oder illegalen Betäubungsmitteln.

In der Zusammenschau der Obduktionsbefunde und der Zusatzuntersuchungen wurde als Todesursache ein Verbluten in die Weichteile infolge massiver stumpfer Gewalteinwirkung festgestellt. Die Verletzungen der linken Gesichtshälfte, des rechten Oberarms und des Nackens (•Abb. 4, 5, 6) waren mit einer Verursachung durch die Borsten des Schrubbers, die geformten Hauteinblutungen der linken hinteren Schulterregion (- Abb. 7) durch die Kanten des Bürstenkörpers vereinbar.

\section{Ermittlungsergebnisse}

Das alte Ehepaar war seit 65 Jahren verheiratet. Vor mehr als 20 Jahren hatte das Paar die spätere Tatwohnung im Erdgeschoss eines Mehrfamilienhauses bezogen. Seit Ende 2017 fiel der demente Ehemann durch eine zunehmende Verwahrlosung mit Vernachlässigung der Körperhygiene und teilweise fremdaggressivem Verhalten auf. Erschwerend kam eine schon länger bestehende Harninkontinenz hinzu. Die Ehefrau reagierte darauf mit verbalen und körperlichen Angriffen gegen ihren Mann. Die Auseinandersetzungen des Ehepaares waren den Mitbe-

Rechtsmedizin 2020 -30:253-259 https://doi.org/10.1007/s00194-020-00398-w

(c) Der/die Autor(en) 2020

I. Wirth · R. Schulz · S. Schmidt · A. Schmeling

\section{Schrubber als Tatwaffe. Tötung des 86-jährigen Ehemanns durch die gleichaltrige Ehefrau}

\section{Zusammenfassung}

Es wird über einen Fall häuslicher Gewalt berichtet, bei dem die 86-jährige Ehefrau ihren gleichaltrigen, an Demenz leidenden Ehemann durch vielfache stumpfe Gewalteinwirkungen tötete. Als Tatwerkzeug kam ein Schrubber zum Einsatz. Richtungweisend für die Identifizierung des Schrubbers als Tatwaffe waren die zahlreichen gruppiert angeordneten, rundlichen, kleinfleckigen Hautabschürfungen sowie die gruppiert angeordneten, kleinfleckigen Hautunterblutungen, die sich den Borstenbüscheln eines Schrubbers zuordnen ließen. Todesursächlich war ein Verbluten in die Weichteile. Eine Fettembolie wurde histologisch ausgeschlossen. In einem Sicherungsverfahren wurde für die Ehefrau aufgrund einer AlzheimerDemenz mit dadurch bedingter mangelnder Steuerungsfähigkeit die Unterbringung in einem psychiatrischen Krankenhaus angeordnet.

Schlüsselwörter

Stumpfe Gewalt · Häusliche Gewalt . Tötungsdelikt $\cdot$ Befundmuster $\cdot$ Obduktion

\section{A scrubber used as a crime weapon. Killing of the 86-year-old husband by the coeval wife}

\section{Abstract}

A case of domestic violence is reported, in which an 86-year-old wife killed her coeval demented husband by inflicting multiple blunt force injuries. A scrubber was used as the weapon. Decisive evidence for the identification of the murder weapon was provided by the numerous grouped, roundish, small patchy excoriations as well as the grouped, small patchy hematomas, which could be correlated with the bristles of a scrubber. The cause of death was exsanguination into the soft tissues. Fat embolism was excluded by histological examination. Due to the wife's diagnosed Alzheimer's disease and therefore diminished accountability, the court ordered confinement in a psychiatric hospital.

Keywords

Blunt force - Domestic violence - Homicide . Pattern of injury · Autopsy wohnern nicht verborgen geblieben. Bis zu den Mietern im 2. Obergeschoss drangen die lautstarken Beschimpfungen des kranken Mannes. Im Sommer 2018 hatte eine Nachbarin zum ersten Mal gehört, wie die Frau schrie: „Ich schlag dich kaputt.“

Eine Zeugin aus der Nachbarwohnung berichtete, dass „der Mann seit August immer wieder von der Frau geschlagen wurde. Man konnte sein, aua, aua immer hören. Ich konnte das Schlagen auch hören. “Bei Begegnungen mit dem Mann fiel den Mitbewohnern mehrmals auf, dass er im Gesicht „richtig große Wunden hatte, die dann verkrustet waren“. Um die Verletzungen zu verbergen, war sein Gesicht manchmal dick mit Creme zugeschmiert oder die Wunden mit Pflaster beklebt.
Die körperlichen Attacken der Ehefrau eskalierten seit dem Sommer 2018. In den folgenden Monaten kam es zu mehreren Polizeieinsätzen wegen häuslicher Gewalt, zuletzt am Tag vor dem Tod des Mannes. Ein anderes Ereignis am Vormittag desselben Tages hatte zu einer Strafanzeige gegen die Ehefrau wegen Körperverletzung gemäß $\$ 223$ StGB geführt. Auf dem Weg zum Gottesdienst hatte eine Passantin beobachtet, dass „die Frau ihren Mann mittels Regenschirm regelrecht traktiert hat“. Die Passantin sprach die Frau an, und es entwickelte sich eine verbale Konfrontation, in deren Verlauf die Passantin von der Frau einen Stoß vor die Brust erhielt. Gegenüber der Polizei äußerte die Passantin ihre Verwunderung darüber, mit welch 


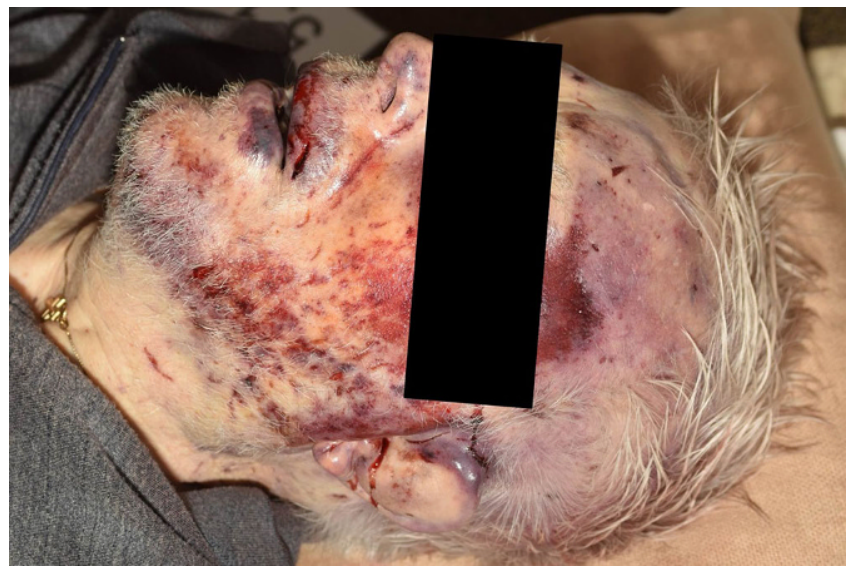

Abb. $4<$ Linke Gesichtshälfte mit bevorzugt gruppiert angeordneten kleinfleckigen Hautabschürfungen

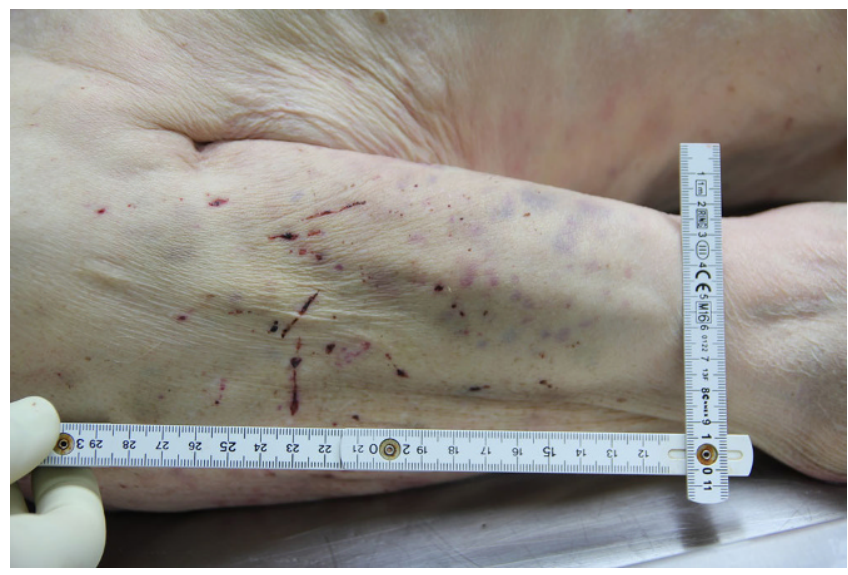

Abb. $5 \triangleleft$ Rechter Oberarm mit gruppiert angeordneten, kleinfleckigen Hautunterblutungen an der Beugeseite



Abb. $6 \triangleleft$ Nackenregion mit kleinfleckigen Hautabschürfungen und Hautunterblutungen

großer Kraft der Stoß ausgeführt worden war.

\section{Strafverfahren}

Am frühen Abend des Tattages wurde die Ehefrau unter dem Verdacht des Totschlags gemäß $\$ 212$ StGB vorläufig festgenommen. Noch am selben Tag sollte sie als Beschuldigte vernommen werden. Bei der Belehrung über ihre Rech- te wirkte sie zerstreut und unterbrach die Beamten dauernd. Zum Tatgeschehen befragt, blieb sie aber hartnäckig bei ihren ursprünglichen Angaben, dass ihr Mann in der Küche gestürzt sei, während sie im Wohnzimmer ferngesehen habe. Ihr Verhalten und auch ihre Antworten auf die Fragen zur Sache ließen den Verdacht aufkommen, dass die Beschuldigte „evtl. geistig eingeschränkt ist". Aufgrund dessen wurde sie im An- schluss an die polizeiliche Befragung in einer Fachklinik untersucht und im Ergebnis nach dem PsychKG stationär eingewiesen. Während des Aufenthalts in der Fachklinik konnte eine weitere psychiatrische Beurteilung der Beschuldigten vorgenommen werden. Die diagnostizierte Demenz und deren Folgen führten zu dem Schluss, dass die Tat im $\mathrm{Zu}$ stand verminderter Schuldfähigkeit im Sinne von $\$ 21$ StGB begangen worden war. Es wurde eine zeitnahe Unterbringung in einer spezialisierten forensischgerontopsychiatrischen Einrichtung auf der Grundlage des $\$ 126$ a StPO zur Sicherung und zur spezifischen Behandlung empfohlen.

Auf Antrag des ermittelnden Staatsanwalts ordnete das Amtsgericht Münster die einstweilige Unterbringung der Beschuldigten gemäß $₫ 126$ a StPO in einem psychiatrischen Krankenhaus an. Im weiteren Verlauf legte der Staatsanwalt eine Antragsschrift im Sicherungsverfahren gemäß $₫ 413$ StPO mit der Begründung vor, dass die Beschuldigte hinreichend verdächtig ist, im Zustand verminderter Schuldfähigkeit und nichtauszuschließender Schuldunfähigkeit einen Menschen getötet zu haben, ohne Mörderin zu sein ( $\$ \$ 212$ Abs. 1, 20, 21, 63 StGB). Er kündigte an, dass in der Hauptverhandlung die Unterbringung in einem psychiatrischen Krankenhaus nach $\$ 63$ StGB beantragt werden wird.

In dem beauftragten psychologischpsychiatrischen Gutachten wurde zusammenfassend festgestellt, dass bei der Betroffenen eine beginnende AlzheimerDemenz gemischter Genese (ICD-10: F00.2) vorliege, die dem Eingangsmerkmal der krankhaften seelischen Störung im Sinne der Schuldfähigkeitsbestimmungen zuzuordnen sei. Aufgrund ihrer krankheitsbedingt beeinträchtigten kognitiven und emotionalen Fähigkeiten sei für das fragliche Tatgeschehen sicher von einer erheblich verminderten Steuerungsfähigkeit gemäß $₫ 21$ StGB auszugehen. Bei Annahme einer das Tatgeschehen auslösenden akuten Überforderungssituation ließe sich aufgrund ihrer krankheitsbedingten mangelnden Affekt- und Impulskontrolle auch eine Aufhebung des Steuerungsvermögens gemäß $₫ 20$ StGB nicht ausschließen. 


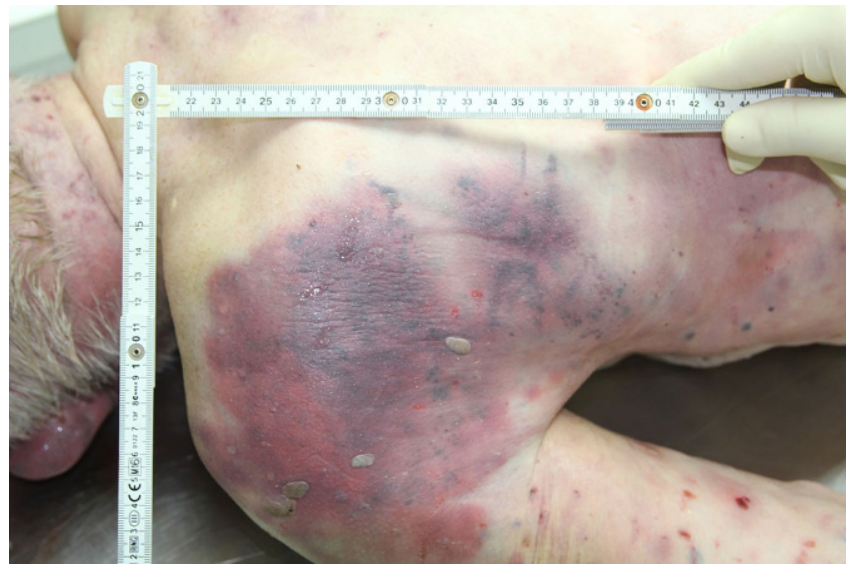

Abb. 7 Linke hintere Schulterregion mit schmalstreifigen, rechtwinkligen Hauteinblutungen inmitten von großflächigen Hautunterblutungen

Aufgrund ihres überdauernden Zustands seien auch zukünftig Gewaltdelikte zu erwarten, sodass die Voraussetzungen einer Unterbringung in einem psychiatrischen Krankenhaus gemäß $\$ 63$ StGB aus gutachterlicher Sicht vorlägen.

Nach 4 Verhandlungstagen ordnete die 2. Strafkammer - Schwurgericht des Landgerichts Münster die Unterbringung der Beschuldigten in einem psychiatrischen Krankenhaus an. In der Urteilsbegründung führte die Kammer aus: „Das festgestellte Geschehen erfüllt die objektiven und subjektiven Tatbestandsvoraussetzungen des $\$ 212$ Abs. 1 StGB, da aber die Steuerungsfähigkeit im Sinne von $\$ 20$ StGB bei Begehung der Tat nicht ausschließbar aufgehoben war, handelte die Beschuldigte ohne Schuld." Das Urteil ist seit dem 17.07.2019 rechtskräftig.

\section{Diskussion}

Die außerordentliche Vielfalt von Schadensereignissen mit stumpfen Gewalteinwirkungen auf den menschlichen Körper bedingt ein beinahe unbegrenztes Spektrum an Verletzungsmustern. Um forensisch verwertbare Rückschlüsse auf den konkreten Entstehungsmechanismus $\mathrm{zu}$ ziehen, ist eine genaue Befundaufnahme mit Erfassung von Verletzungsart, Größe, Form, Farbe, Anzahl und Lokalisation unabdingbar. Obwohl die meisten Verletzungen durch stumpfe Gewalt uncharakteristisch gestaltet sind, können sich im Einzelfall brauchbare Anhaltspunkte für ihre Verursachung ergeben.

Eine Abschürfung mit Verlust oberflächlicher Hautschichten entsteht $\mathrm{zu}$ - meist infolge tangentialer oder schräger Einwirkung auf die Körperoberfläche. Kriminalistisch bedeutsam ist die Möglichkeit, an den zusammengeschobenen Oberhautschüppchen (sog. Epithelmoräne) die Schürfrichtung zu erkennen. Aufgehäufte Hautschüppchen an einem Wundrand belegen eine Abschürfung in Richtung dieses Randes, wenn sich der schürfende Gegenstand auf der Körperoberfläche entlang bewegt hat. Beim Rutschen oder Schleifen des menschlichen Körpers sammeln sich die Hautschüppchen entgegengesetzt zur Bewegungsrichtung. In Abhängigkeit von der Oberflächenbeschaffenheit des schürfenden Gegenstandes kann es zu Materialübertragungen zwischen Gegenstand und Haut kommen (z. B. Erde, Fahrbahnbestandteile, Lacksplitter).

Nur selten entstehen geformte Hautabschürfungen, die den verletzenden $\mathrm{Ge}$ genstand erkennen lassen. Charakteristische Schürfungsmuster sind bei Unfällen jeder Art, speziell im Straßen- und Schienenverkehr, zu erwarten [6-8]. Bei einem Tötungsdelikt konnten kreisrunde Verletzungen im Gesicht dem Rand eines Uhrgewichts zugeordnet werden, mit dem das Opfer durch etwa 15 Schläge getötet worden war [9]. In einem weiteren Fall war eine flächenhafte, parallelstreifige Hautabschürfung in der rechten Stirnregion der getöteten Frau entstanden [9]. Dazu schrieben die Autoren: „Die eigenartige streifenförmige Verletzung an der Stirn der Leiche kam dadurch zustande, daß sie mit der Stirn auf eine Gummiplatte aufschlug und vermutlich noch dadurch stärker abgestempelt worden ist, daß sie, während sie mit der Stirn auf dieser Platte lag, den Schlag mit dem Kochtopf erhielt.“

Anhand von Hämatomen lassen sich nicht nur Art und Schwere der Schädigung, sondern bei wiederholten Gewalteinwirkungen in Abhängigkeit von der Überlebenszeit auch Hinweise auf die zeitlichen Verhältnisse ableiten. Diagnostisch nicht minder bedeutsam ist es, dass eine Hautunterblutung die Form des verursachenden Gegenstands anzeigen kann. Häufige Entstehungsursachen geformter Blutergüsse sind Schläge mit Gegenständen, Fußtritte und Unfälle mit Fahrzeugen. In einer Zusammenstellung spezieller Formen stumpfer Gewalt sind einige markante Beispiele dargestellt [5]. Dazu zählen geformte Hämatome durch Schläge mit einem Stock, einem Queue, einem Baseballschläger und einer Hundekette. Bei den demonstrierten Stockschlägen sind die richtungweisenden Doppelstriemen deutlich ausgeprägt. Im Unterschied dazu hinterlassen flächenhafte Gewalteinwirkungen wie Fußtritte oder Überrollen durch Lkw ausgedehnte Blutergüsse, die an der Haut das Profil des verletzenden Schuhwerks oder Reifens wiedergeben können. Wenn nach massiven Gewalteinwirkungen unter äußerlich sichtbaren Hämatomen ausgedehnte Wundtaschen liegen, sind darin bisweilen große Blutmengen und Gewebetrümmer enthalten.

Insbesondere Schläge mit stumpfkantigen Gegenständen können charakteristisch geformte Quetsch-Riss-Wunden verursachen. Ein markantes Beispiel sind die Kopfwunden durch Schläge mit einem Hammer, der abhängig vom Auftreffwinkel verschiedenartige spezifische Wundformen verursacht $[1,8]$. Kasuistisch wurde über mehr oder weniger deutlich geformte Hautwunden nach Schlägen mit einem Schürhaken [10], mit einem Glasaschenbecher [9] sowie mit Hammer und Meißel [5] berichtet. Besonders präzis konturiert war die Form einer Gesichtsverletzung bei einem Tötungsdelikt durch Erschlagen mit einem Plätteisen. Die Tatwaffe ließ sich in die Verletzung "gut hineinpassen“ [9]. Allerdings muss dieser Befund als Ausnahme angesehen werden, denn je nach Art der Wunde ist eine Abweichung von mehreren Millimetern zwischen der 
Größe der Verletzung und der Wirkfläche des Tatwerkzeugs zu erwarten.

Eine Besonderheit des hier vorgestellten Falles besteht darin, dass am Opfer mehrere Verletzungsarten durch stumpfe Gewalt, von Hautabschürfungen über Hämatome bis zur Quetsch-RissWunde, feststellbar waren. Das außerordentlich vielgestaltige Verletzungsbild resultierte zum einen aus der über lange Zeit verübten Gewalt gegen das Opfer und zum anderen aus den offensichtlich unterschiedlichen Begehungsweisen der Tathandlungen. Die Analyse der Einzelbefunde ließ darauf schließen, dass die Täterin mit dem Schrubber sowohl geschlagen als auch gestoßen hat. Richtungweisend für die Identifizierung des Schrubbers als Tatwaffe waren die zahlreichen gruppiert angeordneten, rundlichen, kleinfleckigen Hautabschürfungen, die durch die Borstenbüschel verursacht worden waren. Die einzelnen Abschürfungen waren auf der linken Gesichtsseite über eine angedeutet rechteckige Fläche weitgehend regelmäßig verteilt. Ein ähnliches Flächenmuster zeigte sich am rechten Oberarm. Hier fanden sich neben Hautabschürfungen auch gruppiert angeordnete, kleinfleckige Hautunterblutungen, da diese Region zum Zeitpunkt der Gewalteinwirkungen bekleidet war. In der linken hinteren Schulterregion zeichneten sich mehrere rechtwinklige Hauteinblutungen ab, die durch Schläge mit einer Seite des Bürstenkörpers entstanden waren.

Über ein vergleichbares Tatgeschehen wurde nur ausnahmsweise berichtet. Eine einschlägige Kasuistik hat Liman im Jahr 1889 veröffentlicht [4]. In diesem Fall war der Verstorbene 10 Tage vor seinem Tod von einem anderen Mann durch „Schläge mit einem Schrubber gegen den Kopf " misshandelt worden. Dabei waren dem Opfer 4 Hautwunden „mit zerrissenen, unregelmäßigen, stumpfen Rändern" zugefügt worden. Die Wunden infizierten sich, und der Tod trat infolge einer Pyämie ein. Das amtseidliche Gutachten endete mit der Feststellung, ,dass diese tödtlich gewordene Krankheit mit den ihm zugefügten Kopfverletzungen ursächlich im Zusammenhang stehe“.

Bei einem Todesfall durch Zyanidvergiftung fand sich als Nebenbefund am
Hinterkopf des Verstorbenen „eine $3 \mathrm{~cm}$ lange zackige, jedoch nur $4 \mathrm{~mm}$ tiefe, also oberflächliche Verletzung" [11]. Im Rahmen der polizeilichen Ermittlungen stellte sich heraus, dass die Frau ihrem Mann bei einer tätlichen Auseinandersetzung die Kopfwunde durch einen Schlag mit dem Schrubber beigebracht hatte.

In einem kurzen Fallbericht über die Tötung einer Frau wurde auch das Schlagen mit einem Handbesen als Begehungsweise erwähnt [9]. Der Täter hatte sein Opfer außerdem von einer Treppe hinabgestürzt. An der Leiche waren großflächige Hämatome an Kopf, Rumpf und Armen erkennbar; geformte Verletzungen ließen sich nicht abgrenzen. Die Todesursache war eine massive Fettembolie.

\section{Fazit}

Über tödliche stumpfe Gewalteinwirkungen mit einem Schrubber liegen in der rechtsmedizinischen Fachliteratur nur sehr wenige kasuistische Mitteilungen vor. Nach unserer Kenntnis wurde das charakteristische Verletzungsbild, das durch Borstenbüschel eines Schrubbers an bekleideter und unbekleideter Haut verursacht wird, bislang nicht beschrieben. In dem gegenständlichen Fall fanden sich an der Haut eines Verstorbenen gruppiert angeordnete, rundliche, kleinfleckige Hautabschürfungen sowie gruppiert angeordnete, kleinfleckige Hautunterblutungen, die sich den Schrubberborsten zuordnen ließen. Bei einem derartigen Befundmuster sollten Stöße mit einem Schrubber in die differenzialdiagnostischen Überlegungen zur Hergangsrekonstruktion einbezogen werden.

\section{Korrespondenzadresse}

\section{Prof. Dr. med. A. Schmeling}

Institut für Rechtsmedizin, Universitätsklinikum Münster

Röntgenstr. 23, 48149 Münster, Deutschland andreas.schmeling@ukmuenster.de

Danksagung. Die Autoren danken dem zuständigen Dezernenten der Staatsanwaltschaft Münster für die freundliche Unterstützung bei der Recherche für die vorliegende Publikation.

Funding. Open Access funding provided by Projekt DEAL.

\section{Einhaltung ethischer Richtlinien}

Interessenkonflikt. I. Wirth, R. Schulz, S. Schmidt und A. Schmeling geben an, dass kein Interessenkonflikt besteht.

Für diesen Beitrag wurden von den Autoren keine Studien an Menschen oder Tieren durchgeführt. Für die aufgeführten Studien gelten die jeweils dort angegebenen ethischen Richtlinien.

Open Access. Dieser Artikel wird unter der Creative Commons Namensnennung 4.0 International Lizenz veröffentlicht, welche die Nutzung, Vervielfältigung, Bearbeitung, Verbreitung und Wiedergabe in jeglichem Medium und Format erlaubt, sofern Sie den/die ursprünglichen Autor(en) und die Quelle ordnungsgemäß nennen, einen Link zur Creative Commons Lizenz beifügen und angeben, ob Änderungen vorgenommen wurden.

Die in diesem Artikel enthaltenen Bilder und sonstiges Drittmaterial unterliegen ebenfalls der genannten Creative Commons Lizenz, sofern sich aus der Abbildungslegende nichts anderes ergibt. Sofern das betreffende Material nicht unter der genannten Creative Commons Lizenz steht und die betreffende Handlung nicht nach gesetzlichen Vorschriften erlaubt ist, ist für die oben aufgeführten Weiterverwendungen des Materials die Einwilligung des jeweiligen Rechteinhabers einzuholen.

Weitere Details zur Lizenz entnehmen Sie bitte der Lizenzinformation auf http://creativecommons.org/ licenses/by/4.0/deed.de.

\section{Literatur}

1. Bratzke H (2015) Stumpfe Gewalt. In: Madea B (Hrsg) Rechtsmedizin, 3. Aufl. Springer, Berlin Heidelberg, S210-215

2. Bundeskriminalamt (2018) Partnerschaftsgewalt - Kriminalstatistische Auswertung - Berichtsjahr 2018. https://www.bka.de/ SharedDocs/Downloads/DE/Publikationen/ JahresberichteUndLagebilder/ Partnerschaftsgewalt/Partnerschaftsgewalt 2018.html.Zugegriffen: 17. Apr. 2020

3. Kolbe V, Büttner A (2020) Häusliche Gewalt gegen Männer - auch ein rechtsmedizinisches Problem? Rechtsmedizin 30:88-93

4. Liman C (1889) Johann Ludwig Casper's Handbuch dergerichtlichen Medicin, 8. Aufl. Bd. 2, Thanatologischer Theil. Hirschwald, Berlin, S312-314

5. Mattig W (2004) Spezielle Formen der stumpfen Gewalteinwirkung. In: Brinkmann B, Madea B (Hrsg) Handbuch gerichtliche Medizin 1. Springer, Berlin, Heidelberg, S526-536

6. Mattig W, Semmler J (2004) Stumpfe Gewalt. Hautund Weichteile. In: Brinkmann B, Madea B (Hrsg) Handbuch gerichtliche Medizin 1.Springer, Berlin, Heidelberg, S358-368

7. Mueller B (1975) Schädigungen und Todesfälle durch Einwirkung von stumpfer Gewalt. In: Mueller B (Hrsg) Gerichtliche Medizin, Teil 1, 2. Aufl. Springer, Berlin, Heidelberg, New York, S385-394

8. Prokop O (1975) Einwirkung von stumpfer Gewalt. In: Prokop 0, Göhler W (Hrsg) Forensische Medizin, 3. Aufl. Volkund Gesundheit, Berlin, S179-183

9. Prokop O, Radam G (1992) Atlas der gerichtlichen Medizin, 3. Aufl. Ullstein Mosby, Berlin, S351-369 
10. Schulz E (1986) Verletzungen durch stumpfe Gewalt. In: Forster B (Hrsg) Praxis der Rechtsmedizin. Thieme, Stuttgart, New York; Beck, München,

S83-87

11. Stoermer R(1928) Zur Kasuistik der Cyankalivergiftung. Dtsch Zges gerichtl Med 12:251-258

\section{e.Medpedia: Die Online-Enzyklopädie für Ärzte}

e.Medpedia ist die neue digitale Enzyklopädie für Ärzte und ermöglicht das schnelle Nachschlagen auf Basis medizinischer Standardwerke von Springer. Die über Peer-ReviewVerfahren begutachteten Einträge werden von über 2.800 erfahrenen klinischen Experten verfasst und fortlaufend aktualisiert.

- e.Medpedia enthält alle Inhalte von über 20 etablierten Referenzwerken von Springer

- Inklusive unzähliger Abbildungen, klinischer Bilder, Tabellen und Schemata sowie Videos

- Verfasst von über 2.800 renommierten Fachärzten, gesichert durch Peer Review-Verfahren

- Komfortable Suchfunktion mit schneller Erkennung der Suchwörter

- Über 7.000 Querverlinkungen zwischen den einzelnen Einträgen

- Die bestehenden Einträge werden fortlaufend aktualisiert

- Weitere Fachgebiete werden kontinuierlich erweitert

- Mobile Nutzung über Smartphones - online und offline mit der e.Medpedia App für iOS- und Android-Geräte

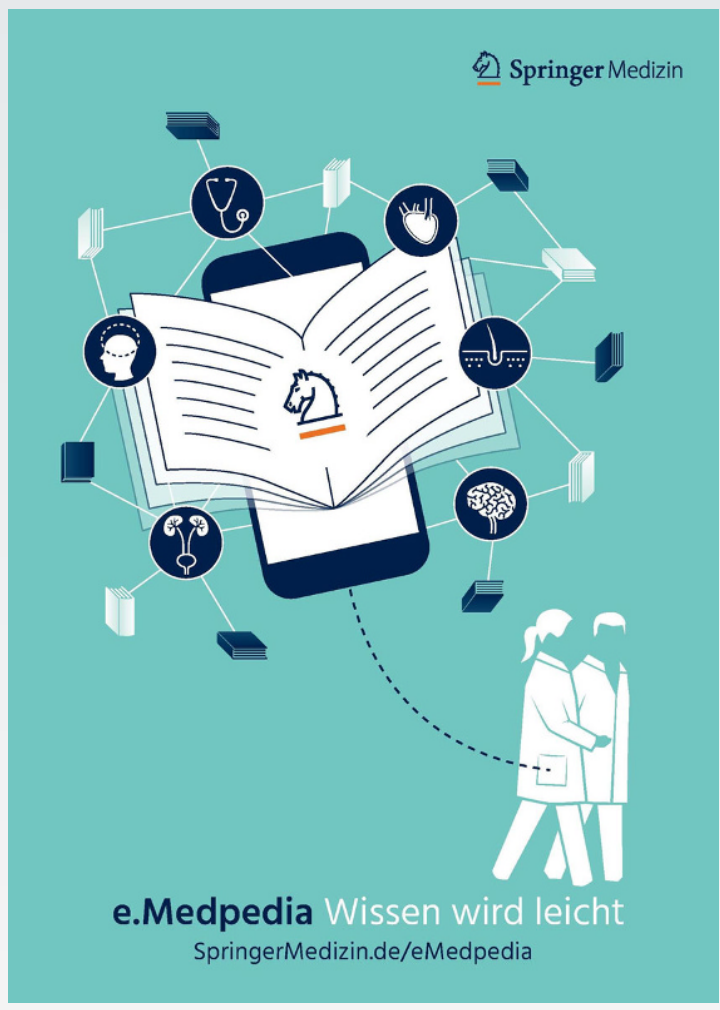

